Voix et Images

voixetimages

\title{
Concomitances et coïncidences dans Bonheur d'occasion
}

\section{Novella Novelli}

Volume 7, numéro 1, automne 1981

Adrien Thério

URI : https://id.erudit.org/iderudit/200308ar

DOI : https://doi.org/10.7202/200308ar

Aller au sommaire du numéro

\section{Éditeur(s)}

Les Presses de l'Université du Québec

\section{ISSN}

0318-9201 (imprimé)

1705-933X (numérique)

Découvrir la revue

\section{Citer cet article}

Novelli, N. (1981). Concomitances et coïncidences dans Bonheur d'occasion.

Voix et Images, 7(1), 131-146. https://doi.org/10.7202/200308ar d'utilisation que vous pouvez consulter en ligne.

https://apropos.erudit.org/fr/usagers/politique-dutilisation/ 


\title{
Concomitances et coïncidences dans Bonheur d'occasion
}

\author{
par Novella Novelli
}

La crise des années 30 met en évidence les problèmes que la société canadienne-française affrontait depuis longtemps. On constate la présence de milliers d'ouvriers sans travail qui, cédant au mirage de la ville et à l'espoir de se sauver de la misère, avaient quitté la campagne ${ }^{1}$ par vagues, particulièrement vers la fin du XIXe siècle, au cours de la seconde industrialisation des années 10 et après la guerre de 14-18. «A mesure que le Québec s'industrialise, ils s'établissent à Montréal, Québec, Sherbrooke, Trois-Rivières. A Montréal en particulier, ils forment autour de la cité anglaise une ceinture de faubourgs [...] autant de villages où refleurissent les institutions rurales faute de participation authentique à la vie urbaine. Comme à la campagne, les activités sont polarisées par la paroisse et la famille ${ }^{2}$." Leur urbanisation, donc, déjà difficile à cause de leur mentalité campagnarde, se complique d'un problème ethnique: "l'arrivée des industries nouvelles représente une invasion par des agents armés du capital et des techniques des centres financiers et industriels plus anciens de Grande-Bretagne et des États-Unis. Ces gérants et techniciens très capables sont étrangers par la culture au milieu canadien-français. Ils le sont toujours par la langue et tempérament, presque toujours par la religion [...] Ainsi le Canadien français devenu ouvrier et citadin se trouve en face d'un patron étranger. Il devient partie d'un système dont l'esprit est anglo-américain plutôt que français ${ }^{3}$."

Gabrielle Roy, en découvrant la misère du quartier Saint-Henri, a perçu le drame de l'intégration ${ }^{4}$ de son "peuple" à la ville: "C'étaient mes frères de sang et de langue que je découvrais, ils vivaient dans une condition d'inférieurs, dans une atmosphère de seconde zone par rapport aux Canadiens anglais ${ }^{5}$."

Dans Bonheur d'occasion 6 , deux générations sont aux prises avec ce problème: la première, celle de Rose-Anna et d'Azarius, est issue de la campagne. Elle véhicule des habitudes et des traditions qu'elle cherche à reproduire à la ville. La deuxième, celle des jeunes: Florentine, Jean, Eugène, bien qu'ils soient nés à la ville, n'y appartiennent pas encore. II y a une troisième 
génération, celle qui a toujours vécu à la campagne: les parents de Rose-Anna. À partir de là, deux intrigues se déroulent en même temps: celle de Florentine et celle de Rose-Anna dont Gabrielle Roy dira: "Cette petite femme du peuple, douce et imaginative, je peux bien vous avouer aujourd'hui qu'elle s'est introduite presque de force dans mon récit, qu'elle en a bouleversé la construction, qu'elle en est arrivée à la dominer par la seule qualité si peu littéraire de la tendresse ${ }^{7}$. L'auteur lui consacrera presque la même place que sa fille: neuf chapitres décrivent son histoire contre les douze pour Florentine. Elle devient le personnage principal, comme l'a bien vu André Brochu: "On s'est souvent demandé qui, de Florentine ou de Rose-Anna, était le personnage principal: ils le sont tous les deux, dans ce qui les oppose (refus de la misère, qui est refus de la maternité chez Florentine) et ce qui les unit (destin commun, legs héréditaires de la misère et de la maternité) ${ }^{8}$ ".

Notre analyse portera seulement sur les chapitres concernant les deux femmes dont les vies sont parallèles et qui, tout en appartenant à deux mondes différents, sont à la même recherche du bonheur. Pour Rose-Anna, il s'agit de défendre un bien acquis: la famille. Attachée au passé, elle lutte contre la désintégration de son foyer. Elle aura le pire destin: spectatrice impuissante, elle assistera aux départs d'Eugène, de Florentine, d'Azarius, et à la mort de Daniel: à cela s'ajoute l'évanouissement de ses illusions. Pour Florentine c'est la conquête d'un monde auquel elle ne s'est pas encore intégrée: la ville, personnifiée par Jean à qui elle ne veut pas renoncer. Ses efforts sont tendus vers l'avenir. Dans cette lutte elle perdra sa famille, sa jeunesse, ses rêves et devra se contenter d'un «bonheur d'occasion".

Si l'histoire de Florentine occupe les deux premiers chapitres, celle de Rose-Anna ne commence qu'au cinquième. Dès que cette dernière s'introduit dans le récit, il est possible de déceler une constante dans le développement des intrigues: un chapitre concerne Rose-Anna, un autre Florentine et un troisième voit la rencontre des deux femmes. L'auteur adopte une technique de concomitances et coincidences qui se poursuit ponctuellement tout au long du roman. Un phénomène qui se produit dans une histoire a des répercussions dans l'autre et transforme les deux univers. Les concomitances nous permettent de suivre l'évolution des deux femmes qui essaient d'accomplir leur intégration à la ville en même temps mais chacune de son côté. Ce qui nous donne la possibilité de comparer les deux mondes, de mesurer la valeur des tentatives des personnages et de comprendre le résultat de leurs efforts. résultat de leurs efforts.

Les coïncidences qui interviennent, c'est-à-dire quand Florentine et Rose-Anna vivent les mêmes événements, nous montrent la séparation des deux univers. Elles ne sont qu'une vérification de leur conquête; les deux femmes, en effet, se rendent compte de l'aboutissement de leurs démarches. Les malheurs ou les joies sont répartis selon l'âge et les personnages.

Le jour où commence la désunion familiale de Rose-Anna, naît en Florentine le faux espoir de s'élever au-dessus de sa condition grâce à Jean. 
La joie que la mère éprouve à la nouvelle du départ pour la campagne, correspond à celle de sa fille qui arrache à Jean la promesse d'une visite. Mais les deux femmes, cédant à leurs illusions, subiront un malheur en même temps : elles perdront leur bonheur. C'est à la suite de la même nuit d'angoisse qu'elles retrouveront le courage de recommencer; "la mère et la fille sont [...] instigatrices de leur propre salut. Nous remarquons cependant que si Florentine et Rose-Anna sont responsables de leur salut comme de leur échec, elles ne le sont qu'en partie: Jean Lévesque et Azarius collaborent à la déchéance, Emmanuel et de nouveau Azarius (cette fois transformé), au salut ${ }^{9}$ ".

Quand les deux intrigues coïncident, l'attitude des personnages change de plus en plus. Florentine, d'abord, véritable soutien matériel et moral de Rose-Anna, prend peu à peu ses distances à l'égard du monde de sa mère, jusqu'à s'en éloigner complètement pour accomplir, dans la solitude, son intégration à la ville. Les chapitres $V$ et $V I$, point de départ de notre analyse, révèlent l'existence des premières concomitances et nous montrent déjà les deux mondes en transformation.

C'est le soir: pendant que Rose-Anna est à la maison et attend, comme d'habitude, ceux qui ne sont pas encore rentrés, Florentine est au restaurant avec Jean. Un événement inattendu se produit dans leur vie: Rose-Anna va perdre son fils qui vient de s'enrôler; Florentine sort pour la première fois avec le jeune homme et son enchantement commence. A l'étourdissement qui suit la nouvelle de l'enrôlement d'Eugène: «Rose-Anna chancela. Pendant une seconde, tout vacilla autour d'elle [...]. Sa voix tremblait, incrédule. Elle n'arrivait pas à formuler les mots qui lui éclataient dans la tête 10 , correspond le "tourbillon» qui soulève Florentine après les baisers de Jean: «tâtonnant dans le vide comme une aveugle, elle gagna le seuil de sa maison [...], elle commença tout de suite à se dévêtir [...] attentive au bourdonnement de son cœur, craignant surtout de rompre le souvenir qui la tenait ${ }^{11}$.

Les deux femmes s'engagent dans la lutte pour le bonheur. La mère, en essayant, par tous les moyens, de dissuader son fils, cherche à le défendre. Rose-Anna "se pencha vers lui et ses yeux brillèrent de détermination [... ] tu vas te dédire. Il est pas trop tard pour te dédire [...] tu leur diras que tu savais pas ce que tu faisais, qu'on a besoin de toi... J'irai, moi, si tu vèux. J'irai, j'expliquerai... ${ }^{12}$ ». La fille veut conquérir Jean, en l'attirant chez lui : «ll faut que je m'arrange pour l'inviter à la maison ${ }^{13} \%$.

Pour Rose-Anna c'est la désunion qui commence. Face à la détermination de son fils "elle semblait vieillie soudain et prête à s'écrouler [...] Sur ses joues amaigries coulaient des larmes vertes comme son visage [...] S'essuyant les yeux, elle gagna le fond de la pièce double et se jeta tout habillée sur son lit ${ }^{14}$ ». Pour Florentine, c'est le début de l'illusion d'accéder à un monde différent. Elle ne doute pas de l'amour de Jean «ll ne me hait pas, s'était-elle dit. Il veut être mon ami de garçon ${ }^{15}$ ». Dès ce moment, elle n'aura qu'un but: se l'attacher. 
Chapitres VII et VIII. Le matin suivant voit les deux femmes sortir de la maison. Rose-Anna contrainte de quitter son logement, comme tous les printemps, part en quête d'un autre. Florentine va travailler au "Quinze-Cents". La mère cherche un logis où elle trouverait le bonheur depuis trop longtemps menacé. Ses démarches, en effet, sont devenues de plus en plus pénibles: «plus la famille avait été nombreuse, plus leur logement était devenu étroit et sombre ${ }^{16}$,.

La fille, qui sert Jean et Emmanuel au restaurant, essaie d'attirer davantage le jeune homme en encourageant l'admiration de son ami. Pour atteindre ce but elle n'ose même pas se venger des méchancetés de Jean par crainte de le perdre: «s'il allait s'éloigner d'elle et ne plus jamais revenir ${ }^{17}$ ».

Après la visite des maisons, Rose-Anna, lasse et découragée, se réfugie dans une église, afin de récupérer ses forces. Elle entretient à nouveau l'espoir de trouver un bon logis, «elle commence par imaginer une petite pièce qui aurait des fenêtres au sud, où elle pourrait installer sa machine à coudre. Puis le soleil gagna la salle à manger; il effleura l'entrée de la cuisine; il y entra ${ }^{18_{\text {}} \text {; }}$ mais, prisonnière du passé, elle est incapable de se tourner vers l'avenir: cette maison dont elle rêve est celle de sa jeunesse.

Après le départ de Jean et Emmanuel, Florentine, qui a été à la fois humiliée, irritée et angoissée, retrouve l'espoir grâce à Emmanuel. Les yeux fermés sur la réalité, elle est décidée plus que jamais à ne pas renoncer à son bonheur. Jean «était peut-être entré dans sa vie afin qu'elle vît bien [...] Jean c'était celui qu'il fallait suivre, jusqu'au bout, pour toujours. Jamais elle ne le laisserait s'échapper [...] jamais elle ne renoncerait à se faire aimer de Jean. Jamais elle ne s'y résignerait ${ }^{19}$ ». Elle rêve donc à la soirée chez Emmanuel où, habillée de sa jolie robe noire, recherchée par tous les jeunes gens, elle sera irrésistible aux yeux de Jean. Sûre d'un meilleur avenir «les mains plongées dans l'eau de vaisselle, elle chantonnait, car elle n'était plus Florentine la serveuse que sa besogne irritait et humiliait profondément [...] elle était Florentine, cette inconnue d'elle-même, un personnage qui lui plaisait, qu'elle avait libéré le soir où follement elle était accourue à la rencontre de Jean dans la tempête - oh! comme elle se plaisait maintenant dans tout ce qu'elle avait entrepris ${ }^{20}$.

Les chapitres $X$ et $X \mid$ concernent Florentine, la deuxième partie du XIle est consacrée à Rose-Anna. Les deux femmes, chacune de leur côté, sont aux prises avec des problèmes propres à leur âge: Florentine éprouve ses premiers chagrins d'amour: Rose-Anna encore une fois lutte contre l'irresponsabilité d'Azarius. Ce qui les rapproche est leur détermination à poursuivre leur objectif. Florentine, mème si Jean n'est pas venu la chercher pour l'emmener chez Emmanuel, est résolue à ne pas manquer cette fête qui lui donne la chance de le revoir. Une fois qu'elle s'aperçoit qu'il n'est pas parmi les invités, le doute commence à s'insinuer en elle: «il n'était pas venu... exprès... Pour ne pas la revoir ${ }^{21}$ " et pour un bref moment, dans l'église, à côté d'Emmanuel, elle cède à la tentation de prier pour que cet amour soit «extirpé» de son cœur. 
Sa déception ne sert qu'à renforcer son attachement à lui. Elle se convainc, qu'elle réussira à le conquérir: "Je me ferais aimer de lui aussi si j'en avais la chance [...] Je le ferais souffrir comme je souffre à cause de lui ${ }^{22}$, elle prie la Sainte Vierge afin qu'elle lui donne la possibilité de le rencontrer.

Rose-Anna, de son côté, essaie de convaincre.son mari de profiter de la chance d'avoir un travail. Devant ses hésitations, elle part elle-même à la recherche de l'ouvrage. Rien ne peut l'arrêter, même pas cette nuit de tempête: c'est son bonheur qui est en jeu. Azarius «croisa le regard de sa femme. C'était le regard grave, énergique, qu'elle avait eu au temps où elle faisait des ménages, cousait pour les autres et, du soir au matin, luttait pour alléger leur misère. Et il baissa la tête ${ }^{23}$ ".

Chapitres XIII et XIV. Une joie arrive en même temps aux deux femmes et leur donne l'illusion d'aller à la rencontre de leur bonheur: pour la mère c'est le départ pour la campagne, pour la fille, la visite de Jean. A la perspective de visiter sa parenté, Rose-Anna est saisie d'une émotion qui lui coupe le souffle. Elle se revoit déjà dans les lieux où elle a vécu une enfance heureuse. La campagne, paradis de ses rêves, seul lieu où elle peut retrouver son bonheur. s'oppose à la ville, à la misère et la rapproche de son mari. «Elle se sentait gagner à la folie d'Azarius. Pour une fois, elle le suivrait dans sa folie, elle qui avait toujours eu le bon sens de son côté ${ }^{24}$. Elle se prépare donc pour le lendemain: "les épaules affaissées, le dos arrondi, les paupières lasses, Rose-Anna cousait pour la fête, en se privant même de chanter pour ne pas effrayer sa joie ${ }^{25}$ ). C'est donc ce soir-là, pendant que Rose-Anna est dans ses préparatifs, que Florentine décide d'aller chercher Jean à la sortie de son travail. Même si depuis trois semaines elle n'a pas de nouvelles de lui, elle n'a pas renoncé à son projet. "Malgré lui, qu'il le voulût ou non, il serait son ami de garçon, son vrai, son steady! Ensemble, ils iraient au cinéma tous les samedis soirs, peut-être deux fois la semaine. Oh, la belle vie qui s'ouvrait devant elle, si seulement elle se montrait assez tenace et pas trop fière pour l'instant! Plus tard, elle saurait bien mener les choses à sa guise ${ }^{26}$. Sûre d'elle et de son pouvoir elle l'invite à la maison pour le lendemain; devant les hésitations du jeune homme à accepter, elle utilise tous les moyens, même les larmes. Son but atteint, "marchant à ses côtés, dans la nuit noire, elle se souriait à elle-même ${ }^{27}$ \%.

Les chapitres XV et XVI constituent le point central de notre analyse et mettent bien en évidence la technique romanesque de l'auteur. Grâce aux concomitances, en effet, les deux générations vivent en même temps le drame de l'intégration. Les personnages, plongés simultanément dans le monde de leurs rêves, la campagne pour Rose-Anna, la ville pour Florentine, ont l'illusion du bonheur à la portée de la main. Pour la mère, il s'agit d'une réintégration à un paradis sacrifié pour un autre plus attrayant, celui de la ville, qui s'est révélé illusoire. Pour la fille c'est l'intégration à la ville à travers Jean. Dès ses premières rencontres elle avait senti ce fort attrait envers le jeune homme: «que cette ville l'appelait maintenant à travers Jean Lévesque! 
À travers cet inconnu, que les lumières lui paraissaient brillantes, la foule gaie [...] Jamais elle n'avait rencontré dans sa vie un être qui portât sur lui tels signes de succès ${ }^{28}$ \%.

Les deux femmes subiront en même temps un échec qui changera leur vie. L'auteur intervient directement dans le récit à travers les «péripéties» provoquées par le hasard: l'accident de voiture pour Rose-Anna et la faute de Florentine marquent un tournant dans leur destinée. C'est en effet une première partie qui se termine et une deuxième qui commence. Rose-Anna perd ses illusions et va à la rencontre de la destruction de son monde; Florentine perd Jean et doit conclure un pacte avec la réalité, celui de se contenter d'un «bonheur d'occasion».

La joie que Rose-Anna éprouvait à la pensée de revoir la campagne de son enfance se révèle éphémère. Il suffit d'un premier contact avec la réalité pour que ses rêves s'estompent. Tour à tour son frère, sa belle-sœur et sa mère la blessent; toute sa misère est devant ses yeux et pour la première fois ses enfants lui apparaissent maigres, les jambes décharnées. Les conseils de sa mère «empreints d'un ton sermonneur, froids comme le visage blanc et anguleux de la vieille femme, se frayaient un chemin à ses oreilles, mais dans son cœur n'éveillaient que le sentiment d'une immense solitude. Qu'était-elle venue chercher exactement? Elle ne le savait plus; car, à mesure qu'elle causait à voix basse avec la vieille, elle oubliait l'image qu'elle s'en était faite à la longue et à distance. Elle la découvrait telle qu'elle avait toujours été, et se demandait comment elle avait pu se leurrer 29 . La campagne donc la rejette, le paradis est perdu à jamais, elle n'appartient plus à ce monde, pas plus qu'elle n'appartient à la ville.

Florentine, qui utilise toutes les coquetteries pour conquérir Jean, ne s'aperçoit pas qu'il est mal à l'aise et qu'il regrette de lui avoir cédé en promettant cette visite. Très souvent un désir de fuite s'empare de lui; cette maison lui rappelle son enfance misérable, la solitude contre laquelle il s'était rebellé. Mais la jeune fille est décidée à aller jusqu'au bout et, dans l'illusion de se l'approprier, se donne à lui. Pour Jean c'est la dernière concession à son passé; en l'embrassant "c'était sa misère, sa tristesse qu'il tenait entre ses bras, sa vie telle qu'elle pourrait être, s'il ne s'était arraché d'elle comme d'un vêtement gênant [...] II se rappelait en même temps que, parfois, dans sa vie, il avait cherché à soulager une peine, [...] II avait encore de ces mouvements généreux. pourvu qu'ils ne gênassent pas la libre expansion de son être. Oui, tout était là; il pouvait à l'occasion céder à la générosité à la condition qu'elle ne lui posât pas d'entraves ${ }^{30}$ ".

Aux tentatives de conquérir le bonheur suit la "punition ${ }^{31}$ " pour les deux femmes qui ont voulu s'élever au-dessus de leur misère. Cette journée, en effet, est un triste présage de malheurs futurs.

Chapitres XVIII, XIX et XXI. Pour Rose-Anna le processus de désagrégation est désormais irréversible et Florentine s'aperçoit qu'elle est la seule victime de son piège. 
L'accident survenu sur la route du retour, Azarius congédié, Daniel malade, la visite à Florentine d'un jeune homme pendant leur absence, c'est à cette série de malheurs, arrivés depuis le voyage à Saint-Denis, auxquels songe Rose-Anna en se rendant à l'hôpital. "Quelle folie que d'être allé aux sucres! Chercher une joie, n'était-ce pas pour eux, est-ce que ce n'avait pas toujours été un sûr moyen de s'attirer la malchance? Oh! qu'elle paraissait absurde et incompréhensible, maintenant, la frénésie de bonheur, qui, tous, les avait saisis ${ }^{32}$ \%. À la tristesse de la maladie de Daniel s'ajoute le sentiment que l'enfant lui échappe. Elle le questionne à propos de son affection envers la jeune infirmière: "tu l'aimes pas mieux que nous autres toujours? ${ }^{33}$ Elle essare de evelller en luı le souvenır de la campagne, de l'école, de la maison: mais tous ses efforts pour se l'attacher sont vains: l'enfant ne désire rien et il n'a pas hâte de rentrer. En sortant de l'hôpital, c'est surtout la pensée de l'avoir perdu qui l'obsède. «Daniel avait tout ce qu'il lui fallait. Jamais il n'avait été si heureux. Elle ne comprenait pas, et elle s'entêtait à chercher une raison. Et un sentiment la saisit à la gorge qui avait le goût du poison. «lls me l'ont pris, lui aussi, pensa-t-elle. C'est facile aussi de me le prendre, il est si petit!» [...] La paix toute nouvelle, toute merveilleuse de Daniel, au lieu de la réjouir, la poursuivait dans l'escalier comme une honte que jamais elle n'oublierait ${ }^{34}$ ". À l'éloignement de Daniel s'ajoute la certitude d'avoir perdu Eugène. Le jeune homme, qui a obtenu quelques jours de congé, l'attend à la maison. II n'a qu'un seul but: lui emprunter de l'argent et sortir tout de suite; «il y avait de la musique, il y avait de la jeunesse dans les rues, il y avait tout cela qui l'attendait ${ }^{35}$ \%. Le foyer de Rose-Anna ne le retient pas, il ne reste même pas pour dîner: "elle lui faisait peur cette maison, avec tous ces rappels de l'enfance. Et la pauvreté qui avait son visage écrit, clairement exprimé, dans chaque recoin! [...] Ah, c'était depuis longtemps qu'il avait voulu fuir ! Et c'était depuis longtemps aussi qu'il était parti ! Et pas pour revenir ! ${ }^{36}$ ». Florentine, qui depuis la visite de Jean n'a plus de nouvelles de lui et doute d'être enceinte, part le chercher. "Elle ne s'avouait pas encore que toutes ses démarches pour retrouver Jean demeuraient vaines. Accablée par son malheur, éperdue de crainte, elle s'imagina que le hasard la servirait ce jour même et que ce soir. sans doute, un miracle se produirait: elle rencontrerait enfin le jeune homme ${ }^{37}$. Bien qu'il lui inspire de la haine, elle voudrait encore qu'il l'aime. Mais une triste surprise l'attend: le jeune homme est parti sans laisser d'adresse. C'est l'évanouissement de ses rêves. Après le premier abattement, le sentiment de recommencer à aimer la vie prend de plus en plus forme en elle: elle songe à tous les vêtements qu'elle aimerait s'acheter. "Ainsi elle se vengerait de Jean. Un sourire d'une incroyable naïveté entrouvit ses lèvres. Elle serait si élégante que, s'il la rencontrait un jour par hasard, il regretterait de l'avoir délaissée. Mais ce serait à son tour à elle de se montrer impitoyable [...] Elle s'évertuerait tellement à mettre dans sa vie toutes les apparences du bonheur, que le bonheur y viendrait faire sa place ${ }^{38^{\prime}}$. Sa vie est planifiée: elle se tournerait vers sa famille: «oui, désormais, elle serait pour sa mère un sûr soutien. Qu'importe qu'Azarius et Eugène ne fissent pas leur part? Elle n'abandonnerait jamais sa mère à leur insouciance [...] La maison allait la reprendre, la guérir ${ }^{39}$ ". 
Chapitres $X X I I I$ et $X X I V$. Selon le même procédé de concomitances, les deux mondes continuent leur évolution: les deux femmes, bouleversées par leur échec, décident, à la suite de la même nuit d'insomnie, de recommencer. Si les efforts de Rose-Anna sont concentrés à recréer son foyer, pour Florentine c'est un chapitre de sa vie qui s'achève. Sa fuite de la maison où elle croyait retrouver la guérison est une fuite d'elle-même et de ses illusions. À l'aube de cette nuit, où elle est chez Marguerite et cherche toutes les issues pour se sortir de sa situation, son cœur, qui avait connu le désespoir et les tremblements convulsifs, était devenu insensible. Le calme s'était fait en elle: «Plus de souvenirs, plus de joie, plus de regret [...] Sa décision était prise. Quoi qu'il en fût, quoi qu'il arrivât, jamais elle ne desserrerait les lèvres sur son secret. Elle se laisserait vivre, elle se laisserait faire, elle attendrait [...] Tout n'était pas fini [...] il devait se produire parfois des miracles, pensa-t-elle, en faveur d'êtres comme elle, fermés et audacieux 40 \%. C'est en renonçant à ses rêves, à son amour, à sa famille et en s'appuyant seulement sur elle-même qu'elle accomplira son intégration à la ville, qui est individualisme et solitude. Le tiède rayon de soleil qui annonce un nouveau jour est pour elle le début d'une nouvelle vie.

Rose-Anna, qui après le départ de sa fille était plongée dans un accablement profond, se réveille de sa torpeur en apprenant qu'Azarius a trouvé un nouveau logis et, en pleine nuit, décide de déménager. Quand elle quitte la cuisine dépouillée, c'est surtout le sentiment du bonheur qu'elle $y$ avait connu qui la retient. Ici Eugène avait dormi, Daniel joué, Florentine tantôt l'avait "supplié des yeux». Dans la nouvelle maison, elle n'arrive pas à dormir, son avenir l'inquiète: cette maison prédisposait-elle au bonheur? avait-elle abrité des gens heureux? «Dans la nuit [...] Rose-Anna se souleva avec précaution et, un coude sur l'oreiller, elle plongea les yeux dans l'obscurité. Elle prêta l'oreille au silence, attentive au mystère de cette maison où ils avaient trouvé abri et qui, cependant, lui restait inconnue ${ }^{41}$ \%. A la découverte des inconvénients «elle ne perdait pas pied. Pas encore. Elle ne renonçait jamais si vite." "ll doit y avoir des avantages avec les désavantages, pensait-elle. C'est au matin que je verrai comme il faut. Là, il faut pas se dépêcher de tout voir en noir ${ }^{42}$. C'est elle qui encore une fois réconforte son mari: «On est encore ensemble, Azarius. On a encore not' force, not' santé. Qu'est-ce que tu veux qui nous arrive de pire ? ${ }^{43}$. Quand il s'endort elle se lève pour explorer toutes les pièces du logis. L'aube la surprend depuis longtemps au travail, agenouillée sur le plancher, dans un courageux effort de recommencement.

Chapitres $X X I X$ et $X X X I$. Les deux femmes, qui en même temps ont décidé de repartir à zéro, auront un sort différent: l'une, en adoptant une solution de rechange, réussira dans ses efforts; l'autre échouera, prisonnière d'un monde qui évolue vers son anéantissement.

Florentine, qui ne reviendra jamais sur ses pas, met en pratique le plan mûri depuis la veille, quand sa mère l'avait mise au courant de la visite d’Emmanuel. Sa décision était prise: ce serait lui qui la sortirait de son état. Pendant toute la journée passée avec lui, elle poursuit son but: lui plaire et 
l'amener à se faire épouser; «elle se tendait dans un suprême effort pour paraître gaie et même attentive à certaines choses qui plaisaient à Emmanuel ${ }^{44}$ ». Consciente qu'elle ne peut pas renoncer à cette chance, elle a peur de le perdre. «Si elle perdait Emmanuel maintenant, tout serait fini entre eux. Tout. Elle serait perdue cette fois complètement [...] C'était plus que sa sécurité, son salut, qui étaient en jeu. Emmanuel, il lui semblait, arriverait peut-être à lui donner le goût de vivre, un nouvel orgueil, de la joie encore à être bien mise, coquette et irrésistible. Par lui elle avait recommencé à se trouver jolie, ardente 45 ». Grâce à ce mariage, elle aura accès à la ville: elle se voit déjà gâtée par Emmanuel, elle pense aux cadeaux qu'il lui offrira, à la lumière des cinémas et des grands magasins.

À Rose-Anna toute possibilité de recommencement est refusée, elle se voit jour après jour privée d'une partie de son bonheur. Son monde ressemble à un bateau à la dérive. Le désordre et le désarroi qui règnent dans sa maison, reflètent la confusion de son âme face aux événements qui s'étaient succédé. Elle n'avait eu ni le temps ni l'envie de s'installer. "Elle se faisait l'effet d'être emprisonnée entre ces quatre murs rien que pour souffrir, pas autre chose ${ }^{46}$ ". A la perte de Florentine qui s'était mariée et était partie sans aucun regret, s'ajoute aujourd'hui même, où elle donne naissance à un nouvel enfant, la mort de Daniel. Pendant son accouchement des visions de sa vie heureuse se mêlent dans son esprit; sa pensée va à ceux qui l'ont quittée: Eugène, Florentine, Daniel. Ses efforts pour lutter contre un destin qui la dépasse sont vains: cet enfant qui vient de naître ne peut ni compenser la perte des autres ni s'opposer à la destruction entamée.

Chapitres $X X X I I$ et $X X X I I I$. C'est le dernier acte. Les deux mondes marchent parallèlement sur des voies différentes jusqu'à la fin. La dernière concomitance marque l'accomplissement des deux destinées. Azarius et Emmanuel, responsables de la vie des deux femmes, partent en même temps. Pour Rose-Anna l'enrôlement de son mari c'est la dernière peine, pour Florentine, au contraire, ce sont les prémices d'un nouvel avenir.

Cette guerre qui apporte le "salut", pour la mère est pire que la paix: elle la prive des membres de sa famille, de son bonheur, et il n'y a pas d'argent qui puisse compenser cette perte. Le prix de la tranquillité économique, que lui laisse entrevoir Azarius, pour Rose-Anna est trop cher: «tu vas pouvoir vivre comme t'as toujours voulu vivre [...] c'est la tranquillité. De la tranquillité comme on n'a jamais eue $!^{47}$. Quand elle voit son mari devant elle, vêtu de l'uniforme militaire, "ses yeux s'ouvrirent, démesurés. Sa bouche frémit. Et soudain elle poussa un grand cri, un seul, qui se perdit dans la marche sifflante d'une locomotive 48 \%. C'est le dernier coup. Son monde s'écroule.

Florentine, grâce à son mariage, aura l'aisance et retrouvera confiance en elle-même. Les jours passés avec Emmanuel ont été l'occasion de profiter du luxe et de la ville: elle essayait mille fois par jour ses nouveaux vêtements, elle voulait toujours sortir, se promener dans les rues et s'attarder à toutes les vitrines. Au premier soulagement qui suit le départ d'Emmanuel, après la 
rencontre avec Jean, elle retrouve l'estime d'elle-même, et le retour de son mari "auquel elle n'avait jamais songé sans effroi lui parut maintenant tout naturel. Sa voie était nette, claire. Elle s'en allait vers l'avenir, sans grande joie, mais sans détresse [...] II n'y avait pas de péché, pas de faute, pas de passé: tout cela était fini. II n'y avait plus que l'avenir [...] Toutes sortes de projets s'offraient à elle [...] Elle voyait les difficultés s'éloigner, très loin, très loin déjà. Ah! oui, c'était vraiment une vie nouvelle qui commençait ${ }^{49}$ ».

Dans les cinq chapitres consacrés aux coïncidences, les deux mondes se séparent de plus en plus jusqu'à devenir étrangers l'un à l'autre. C'est, en effet, en se rencontrant qu'ils se confrontent et prennent un chemin différent. C'est donc grâce aux coïncidences que le récit avance et il est possible de suivre, comme on l'a déjà vu à travers les concomitances, les transformations qui se produisent dans les deux histoires.

Dès la première coïncidence, qui a lieu à la fin du chapitre VI, les deux femmes prennent leur distance et un contraste se produit. Florentine, qui rentre à la maison après sa première sortie avec Jean, n'écoute presque pas les plaintes de sa mère. "Comment les petites misères quotidiennes auraientelles pu désormais la toucher! Et les troublantes confidences de minuit, dans le silence lourd de respirations, les entendrait-elle jamais encore avec son cœeur angoissé d'autrefois? Une vague la berçait qui était souple et longue et ondoyante ${ }^{50}$ ". Les efforts de Rose-Anna pour atteindre sa seule confidente sont vains: elle ne partage pas comme d'habitude ses ennuis, en plus elle manifeste déjà la volonté de se soustraire au sort de sa mère. Son opposition à la résignation de cette dernière est ferme: «Qu'est-ce que tu veux, Florentine, on fait pas comme on veut dans la vie; on fait comme on peut. " «C'est pas vrai, songeait Florentine. Moi, je ferai comme je voudrai. Moi, j'aurai pas de misère comme sa mère ${ }^{51}$ ». Cet engagement et la pensée de Jean, ce soir, ont gagné sur la misère des siens.

Chapitre $I X$. Le processus d'éloignement déjà manifeste dans le chapitre précédent prend de plus en plus consistance. L'auteur, pour mieux faire ressortir le contraste des deux mondes, situe les deux personnages hors de la maison, lieu habituel de leurs rencontres. Rose-Anna qui est partie tôt chercher un nouveau logis, s'arrête au "Quinze-Cents", le restaurant où Florentine travaille. Une heure avant la jeune fille a reçu la visite de Jean et Emmanuel. Sa mère la trouve encore plongée dans ses pensées. Sans l'obscurité de la nuit, sans le foyer domestique pour cacher sa misère, Rose-Anna est sans voiles devant sa fille. C'est comme si Florentine la voyait pour la première fois, "elle et son pauvre sourire qui avait l'air, dès l'abord, de chercher à égarer l'attention, du moins à la détourner d'elle-même. Florentine en était atterrée ${ }^{52}$ ". Le désir de l'aider qui s'empare d'elle est significatif de l'étrangeté qui s'affirme vis-à-vis de sa mère: "soudain, elle comprit pourquoi ce désir inaccoutumé, ce désir à vrai dire inconnu lui gonflait le cœur ; c'est qu'elle apercevait la vie de sa mère comme un long voyage gris, terne, que jamais, elle, Florentine, n'accomplirait; et c'était comme si, aujourd'hui, elles eussent en quelque sorte à se faire des adieux. Peut-être ici même leurs routes étaient-elles en train 
de se séparer ${ }^{53}$. Tous les gestes, toutes les pensées et les paroles de Florentine témoignent de son détachement. Sa générosité envers sa mère lui donne l'illusion d'avoir droit à la récompense d'un meilleur avenir: «je suis bonne pour maman. Ça me reviendra, ça me sera compté ${ }^{54}$ ». Mais en regardant sa mère s'éloigner, elle se rend compte que son acte «était une pure perte [... ] il ne servait à rien. C'était une goutte d'eau dans l'aridité de leur existence ${ }^{55}$ ». Le sentiment de vouloir échapper au destin de sa mère est le seul qui lui inspire cette timide image qui disparaît au loin: «s'échapper, elle seule, de leur vie, c'était déjà beaucoup، c'était déjà très difficile [...] En garde contre l'attendrissement, son cœur déjà se durcissait ${ }^{56}$ \%.

Chapitre XIII. Florentine, qui depuis la visite de Jean au «Quinze-Cents» ne l'a plus revu, passe presque toutes les soirées chez elle. Ce samedi soir de congé elle est à la maison avec sa mère. L'auteur fait vivre aux deux personnages un même événement, provoqué par le hasard: la nouvelle du départ pour la campagne. De leur réaction on aperçoit l'irrémédiable opposition de deux mondes, antinomie que l'on ressent déjà au début du chapitre quand les deux femmes sont dans la même pièce mais déjà très lointaines l'une de l'autre. Rose-Anna est calme et contente: ses enfants dorment, Eugène lui a rendu une courte visite, sa fille est à côté d'elle. Azarius va bientôt rentrer de son travail, elle ne peut rien demander de plus. Florentine, au contraire, est nerveuse, irritable, «elle s'ennuyait dans cette pièce silencieuse. Et plus que l'ennui encore, la haine de ce pauvre logis, comme un clos où venaient mourir toutes leurs tentatives d'évasion, la tourmentait ${ }^{57}$ ». C'est dans cette atmosphère qu'Azarius surprend les deux femmes et leur annonce le voyage à Saint-Denis pour le lendemain. Rose-Anna demeure incrédule: "elle l'arrêta d'un geste, pâlissant d'émotion, de trop de surprises, d'inattendu qui lui gonflaient le cœur ${ }^{58}$ n. Bouleversée par cet événement, elle essaie de revenir à la réalité: "voyons, elle devait être plus raisonnable, ne pas s'abandonner ainsi ${ }^{59} \%$, mais déjà les rêves la transportent dans un autre univers. Florentine, de son côté, "muette, étonnée, hostile, suivait leurs discours ${ }^{60}$ » et à l'invitation de son père répond d'abord simplement: "non, j'y vais pas moi [... ] mais allez-y vous autres. Je garderai la maison ${ }^{61}$ ». Aux insistances d'Azarius : «mais, fifille, dit-il pour la dérider, ca ne te tente donc pas, les sucres, la tire, et de te fairé un beau cavalier à la campagne ${ }^{62}$, son ton devient de plus en plus sec: “Qu'est-ce que tu veux que ca me fasse? dit-elle, allumant une autre cigarette ${ }^{63}$ \%. Sa réponse finale et nette décourage le pauvre homme : «laisse-moi tranquille, dit-elle. Je veux pas y aller à vos sucres, c'est toute ${ }^{64}$. Elle est donc indifférente à leur monde, ce sont «vos sucres», remarque-t-elle, et cette joie qui les envahit tous la gêne à tel point qu'elle sort de la maison chercher son bonheur à elle: Jean.

Chapitre $X X I I$. Toute réconciliation des deux mondes est impossible, leur séparation est désormais définitive; les deux femmes parlent un langage différent et se dressent l'une contre l'autre comme des ennemies. Florentine, qui n'a pas trouvé Jean et comprend qu'elle l'a perdu à jamais, espère se guérir de sa déception en se rapprochant des siens. Cette tentative est le dernier lien 
avec le monde de sa mère, mais c'est une marche en arrière qu'elle ne peut plus accomplir tellement les deux univers ont évolué. Ses bonnes intentions s'effondrent dès qu'elle ouvre la porte de la maison: elle tombe en plein déménagement, tout est désordre, des visages inconnus l'accueillent : le foyer qui devait lui donner la force de recommencer est détruit. «Était-ce donc à cela qu'elle s'attendait lorsqu'elle était revenue à la maison si désireuse de retrouver chaque chose à sa place, et comme un signe infaillible de sa sécurité? ${ }^{65}$ „Sa mère ne perçoit pas son état d'âme et, comme d'habitude, commence à énumérer leurs malheurs: Azarius, Daniel, Eugène..., mais Florentine, sourde à ces appels cent fois répétés, «devant elle ne desserrait pas les lèvres. Des larmes montaient à ses paupières, brûlantes de révolte. Et voilà donc ce qu'elle était venue chercher auprès de sa mère: des malheurs si grands et si nombreux qu'elle sentit s'étouffer en elle les dernières lamentables velléités d'espoir ${ }^{66}$. Devant le malaise de Florentine, Rose-Anna croit deviner sa honte, elle la regarde «avec des yeux agrandis, fixes, et une expression de muette horreur. Sans pitié, sans amitié, sans bonté: rien que de l'horreur plein les yeux ${ }^{67}$ \%. Florentine, qui la première baisse le regard, espérant de l'aide, des conseils, la supplie encore une fois des yeux, mais sa mère déjà avait tourné la tête et plongeait dans un accablement profond: l'incommunicabilité entre elles était désormais insurmontable. Florentine n'a plus rien à faire ici, pas de famille, pas de soulagement à sa peine, son refuge a été une illusion. Elle est seule avec son angoisse et son secret, il ne lui reste que la fuite: elle tourna sur elle-même, ouvrit la porte d'un coup sec et s'enfuit dans une lame de vent qui semblait la happer ${ }^{68}$.

Chapitre $X X X$. La dernière coïncidence n'est que l'accomplissement de la séparation entre Florentine et sa mère. L'impossibilité de se réintégrer à sa famille, amène la jeune fille à comprendre qu'elle ne peut compter que sur ses propres forces. C'est pour cela qu'après avoir revu Emmanuel, qui a obtenu quelques jours de permission et lui propose de l'épouser, elle accepte: il est sa planche de salut. Á l'éloignement moral de Florentine suit maintenant son départ de la maison. C'est le jour de son mariage, Rose-Anna la réveille et l'aide à s'habiller. Les deux femmes, qui depuis leur dernière rencontre ne se regardaient presque plus et encore moins ne se parlaient, sont incapables d'avoir une conversation, elles échangent à peine quelques mots. L'incompréhension établie entre elles est encore une fois soulignée. Maintenant c'est Rose-Anna, qui remarquant le peu d'enthousiasme de sa fille, lui propose de l'aider, mais pour Florentine il est trop tard et comme seule réponse elle lui dit: «laisse-moi faire [...] je suis capable de $m$ 'habiller toute seule ${ }^{69}$. Elle éprouve, pour un bref moment, la tentation de se jeter dans les bras de sa mère («Rose-Anna, s'étant détournée, hésita une courte minute, puis s'en fut dans la cuisine ${ }^{70}$ ). Florentine reste seule dans sa chambre.

La sensation d'étrangeté que Rose-Anna avait ressentie en regardant sa fille dans la glace: «la bouche était dure, le regard volontaire, presque insolent. Elle l'examinait avec stupeur. Cette Florentine, au masque raidi, aux sourcils froncés, lui était une inconnue ${ }^{71}$, devient, maintenant qu'elle part 
de la maison, une certitude. En la voyant s'éloigner «elle agita la main, ce qui, après réflexion, lui parut futile, car la voiture disparaissait déjà, et d'ailleurs Florentine ne regardait point en arrière. Elle n'avait eu en partant aucune hésitation, aucun geste ému, pas même un seul regard un peu prolongé. Elle était partie comme si rien ici ne la retenait plus, ne la touchait plus, pensa Rose-Anna, «quasiment comme une étrangère ${ }^{72}$.

On a suivi parallèlement à travers I'histoire de Rose-Anna et de Florentine le drame de deux générations attirées et repoussées par la ville. Pour atteindre ce but l'auteur a utilisé une technique de concomitances et de coïncidences. Chaque fois qu'il recourt aux concomitances, c'est pour montrer l'évolution des deux mondes, tandis que le principe qui gouverne les coincidences est leur séparation. Pour ce qui concerne encore la technique romanesque on peut diviser le roman en deux parties. La première à peu près vers la moitié, auX chapitres $X V$ et $X V I$, quand l'auteur situe Rose-Anna à la campagne et Florentine à la ville avec Jean. C'est ici que l'on assiste à l'insuccès de leur première tentative. La deuxième, au moment où les deux femmes essaient d'entamer une nouvelle vie, marque la victoire de Florentine et la défaite de sa mère.

Dans la première partie, on s'aperçoit qu'elles agissent presque de la même façon et cela les empêche de parvenir à leurs fins. Elles voudraient accomplir cette intégration tout en préservant leurs valeurs traditionnelles; pas de renoncements, tout à gagner. Pour Rose-Anna la bataille est perdue d'avance. Elle est trop liée à son passé. Ses origines campagnardes l'étouffent. Dès le début l'auteur nous la montre dans son univers : la maison où, incapable de dormir tant que tous ne sont pas rentrés, elle essaie de donner une cohésion à son foyer pour y retenir les siens. Déterminée à poursuivre cet objectif, tour à tour, elle s'oppose à l'enrôlement d'Eugène, part chercher une nouvelle maison ou de l'ouvrage pour son mari, gagne de l'argent en faisant du ménage et de la couture et est attentive aux soucis de sa famille. On ressent toute la portée de son attachement au passé à l'occasion du voyage à la campagne où elle espère retrouver le bonheur refusé par la ville. Mais ses rêves ne sont qu'un moyen d'échapper à la réalité pour ne pas se mesurer avec elle. Florentine part avantagée par rapport à sa mère. En effet, elle est née à la ville, mais les conditions défavorables où elle vit l'empêchent de s'intégrer à ce monde dont elle apprécie tous les attraits. Son attachement à la famille est très fort, elle en supporte le poids matériel et moral en remplissant les fonctions du pater familias. Dans cette première partie, donc, bien qu'elle s'éloigne du monde de sa mère elle ne s'en détache pas complètement. Elle aussi voudrait accomplir ce passage sans rien payer en échange, tout en restant fidèle à sa famille et à ses principes.

Dans la deuxième partie, Rose-Anna, qui a assisté à l'effondrement de ses illusions, continue inflexible dans la même voie. Elle n'a rien appris de ses revers. Tout continue comme avant. La famille est son seul bonheur à défendre. Pour cela ses efforts de recommencement n'aboutiront à rien, son intégration ne s'accomplira jamais et cette famille pour qui elle a lutté toute 
sa vie va bientôt se disperser. La ville avec ses séductions lui ravira ses fils, la privera de son mari. La deuxième tentative de Florentine, par contre, réussira grâce à son mensonge et à sa désolidarisation de la famille. Elle comprend que c'est seulement en s'appuyant sur elle-même qu'elle verra l'accomplissement de ses efforts. La ville est solitude, sacrifice et accommodement. Florentine peut $\checkmark$ accomplir son intégration après en avoir payé le prix.

Dans cette deuxième partie l'auteur met bien en évidence à travers les concomitances et les coïncidences des deux intrigues que seuls les individus et non pas les familles s'intègrent à la ville, comme l'avait compris Jean, et comme Florentine le comprendra : pour cela Rose-Anna est destinée à échouer et sa fille à réussir.

1. Au sujet de la littérature consacrée à la terre, voir les ouvrages suivants: Maurice Lemire, Le Mythe de la terre paternelle, dans Le Merveilleux, Québec, Les Presses de l'Université Laval, "Histoire et sociologie de la culture». 4, 1973, pp. 55-56; $M$. Servais-Maquois, Le Roman de la terre au Québec, Québec, Les Presses de l'Université Laval, 1974; F. Marcoto, "Note sul romanzo quebechense della terra", /l lettore di provincia, Ravenna, $n^{\circ} 31 / 32$ (mars 1978), pp. 76-84.

2. Maurice Lemire, «Bonheur d'occasion ou le salut par la guerre $"$, Recherches sociographiques, vol. $\mathrm{X}, \mathrm{n}^{\circ} 1$ (janvier-avril 1969), p. 24.

3. E.C. Hughes, Rencontres de deux mondes. La crise d'industrialisation du Canada français, traduit par Jean-Charles Falardeau, Montréal, Parizeau, 1945, pp. 20-21.

4. Maurice Lemire, "Bonheur d'occasion ou le salut par la guerre», op. cit.

5. Conversations avec l'auteur, le 21 septembre 1979.

6. Pour les études sur l'œuvre ou la vie de Gabrielle Roy voir: Marc Gagné. Visages de Gabrielle Roy, Montréal, Beauchemin, 1973; François Ricard, Gabrielle Roy, Montréal, Fides, «Écrivains canadiens d'aujourd'hui», 1975.

7. Cf. Gérard Bessette, Une littérature en ébullition. Montréal, Éditions du Jour, 1968 , p. 227.

8. André Brochu, "Thèmes et structures de Bonheur d'occasion", dans Écrits du Canada français, Montréal, Écrits du Canada français, 1966, p. 195.

9. Jacques Blais, “l'Unité organique de Bonheur d'occasion", Études françaises, Montréal, vol. VI, no 1, (février 1970), p. 33.

10. Gabrielle Roy, Bonheur d'occasion, Montréal. Éditions Internationales Alain Stanké, coll. *10/10», 1977, pp. 72-73.

11. Jbid., p. 87.

12. Ibid., p. 73

13. Ibid., p. 86

14. Ibid., pp. 75-76.

15. Ibid., p. 85 .

16. Ibid., p. 96 .

17. Ibid., p. 112

18. Ibid., p. 103

19. Ibid., p. 117

20. Ibid., p. 118.

21. Ibid. p. 137 


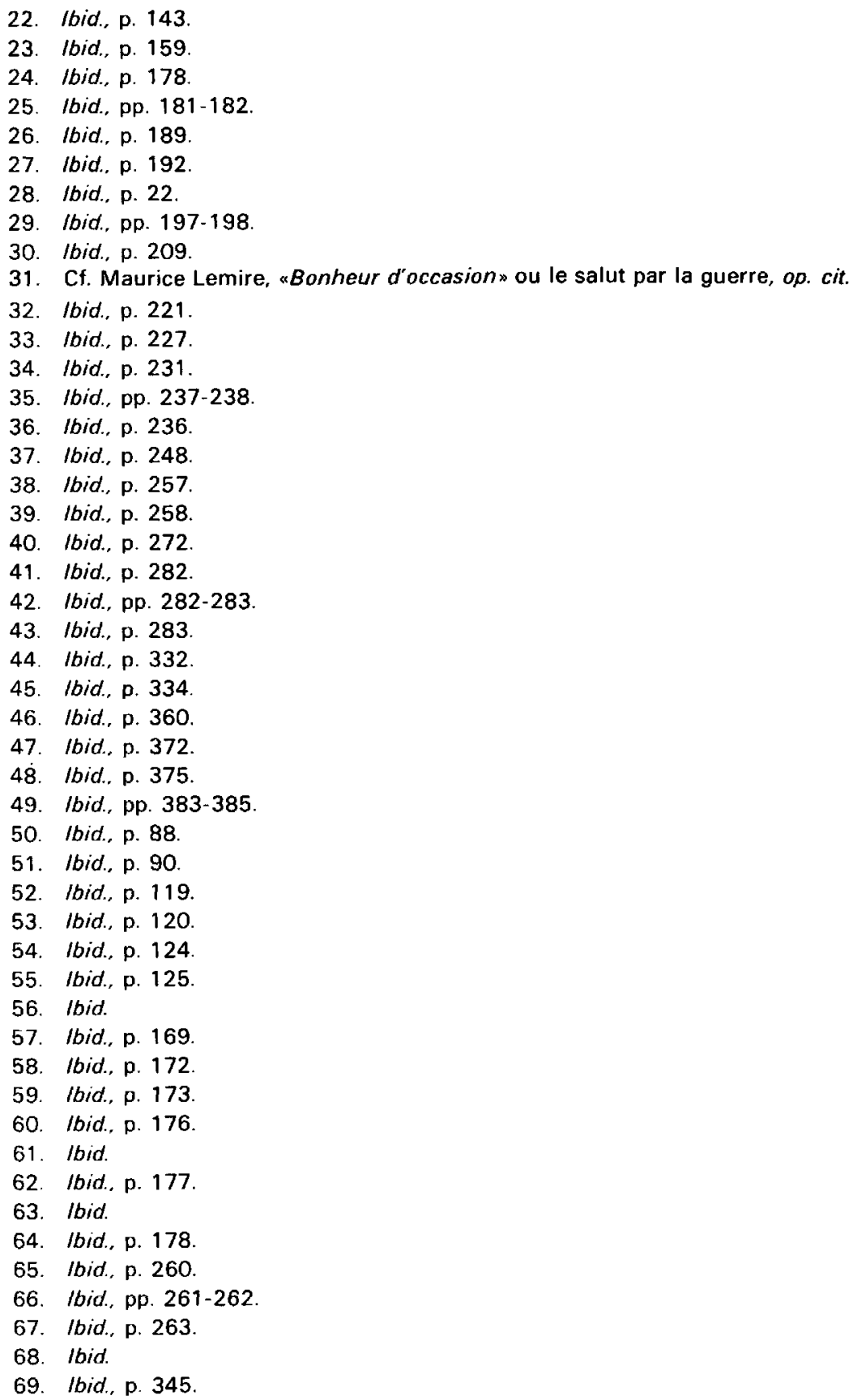


146 VOIX ET IMAGES, VOL. VII, NO 1

70. Ibid., p. 347.

71. Ibid., pp. 345-346.

72. Ibid., p. 351. 